\title{
Influência da calça de compressão no lactato sanguíneo após exercício de agachamento*
}

\author{
Compression pants influence on blood \\ lactate after squat exercise
}

Lia Nara de Moraes Guazzelli ${ }^{1}$ Lucas Pereira Sousa ${ }^{2}$ Márcio Rabelo Mota ${ }^{3}$
Recebido em: 19/09/2016. Aprovado em: 24/01/2017.

UnB - Universidade de Brasília, Brasília, Brasill.

2 UniCEUB - Centro Universitário de Brasília, Brasília, Brasil.

3 UniCEUB - Centro Universitário de Brasília, Brasília, Brasil.

\section{Resumo}

As roupas de compressão aumentam o fluxo sanguíneo favorecendo a ressíntese de ADP com menores níveis de lactato pós-exercício. O objetivo desse estudo foi comparar a concentração de lactato sanguíneo utilizando ou não a calça de compressão no exercício de agachamento. Participaram do estudo 8 sujeitos (21,50 $\pm 3,07$ anos, 79,88 $\pm 12,26 \mathrm{~kg}$ e $1,77 \pm 0,08 \mathrm{~m}$ ). Mediu-se o lactato nos momentos Pré, Pós0’ e Pós10’ do exercício. No Pós0' o lactato apresentou-se elevado comparado com o Pré nas duas condições (experimental 15,10 $\pm 1,25$ e 2,80 $\pm 0,76$; controle 10,41 $\pm 1,27$ e $3,21 \pm 0,80, \mathrm{p}<0,05)$. O Pós0’experimental apresentou lactato, significativamente, mais alto que o controle $(15,10 \pm 1,25$ e 10,41 $\pm 1,27$, $\mathrm{p}<0,05)$. No Pós10’experimental, o lactato se manteve, significativamente, mais alto que no Pré $(12,64 \pm 1,70, \mathrm{p}<0,05)$. Concluiu-se que o uso de calça de compressão foi associado a níveis mais altos de lactato no teste Pós0' e no teste Pós10'.

Palavras-chave: Lactato. Roupa de Compressão. Agachamento. Exercício Resistido.

\section{Abstract}

The use of compression garment increases blood flow favoring the ADP resynthesis with lower levels of lactate post-activity. The aim of this study was to compare the blood lactate concentration before and after the squat exercise, using and not using compression pants. Eight male subjects participated in the study $(21,50 \pm 3,07$ years, 79,88 $\pm 12,26 \mathrm{~kg}$ e $1,77 \pm 0,08 \mathrm{~m}$ ). The lactate concentration was measured in three moments: Pre, Post0' and Post10' of exercise. At Post0' lactate was higher than Pre in both conditions (experimental 15,10 $\pm 1,25$ e 2,80 \pm 0,76; control 10,41 $\pm 1,27$ e 3,21 $\pm 0,80, \mathrm{p}<0,05)$. At Post0'experimental lactate was significantly higher than control $(15,10 \pm 1,25$ e 10,41 $\pm 1,27$, $\mathrm{p}<0,05)$. At Post10'experimental, lactate was significantly higher than at pre $(12,64 \pm 1,70, \mathrm{p}<0,05)$. The use of compression pants was associated with significantly higher levels of lactate in Post0' test and Post10' test. Keywords: Lactate. Compression Garment. Squat. Resistive Exercise. 


\section{Introdução}

A compressão elástica pode ser explicada com a aplicação de uma força em uma área da superfície corpórea. As roupas elásticas terapêuticas indicam existir um perfil de compressão, determinada in vitro, que favorecem a circulação e foram, inicialmente, pensadas para a melhora do retorno venoso em doenças venosas crônicas, principalmente de membros inferiores (FIGUEIREDO; CASTRO; SIMÕES, 2011)

Na prática esportiva, os atletas, sempre, estão em busca de novas formas de melhorar o rendimento, juntamente a uma melhor e mais rápida recuperação muscular após o esforço físico. O efeito benéfico das roupas de compressão chegou à prática esportiva, para que o atleta venha buscar mais conforto nas modalidades praticadas, inclusive com a prática do exercício resistido (FIGUEIREDO; FIGUEIREDO; SILVA, 2011).

Sear et al. (2010) observaram, no seu estudo com roupas compressivas usadas no corpo inteiro durante treinamento intervalado de longa duração, que os avaliados (8 atletas do sexo masculino, 20,6 $\pm 1,2$ anos, 72,9 \pm $5,9 \mathrm{~kg}, 57.5 \pm 3.7 \mathrm{ml} . \mathrm{kg} . \mathrm{min}$ ) percorreram uma maior distância durante o teste, evidenciando uma redução nos níveis de lactato, melhorando a performance e mostrando que a roupa de compressão aumenta o fluxo sanguíneo trazendo benefícios na ressíntese de adenosina difosfato (ADP).

Figueiredo, Figueiredo e Silva (2011) mostraram, em seu estudo, que o uso das meias de compressão, nas diversas modalidades esportivas, como voleibol, bem como atividades físicas de alta intensidade de predominância anaeróbica, possui um benefício indireto, como menores níveis de lactato pós-atividade, menor tempo de recuperação, maior conforto durante a atividade e, consequentemente, performance melhorada.

O lactato é definido como uma mistura orgânica que serve como uma das fontes de energia consumidas pelo corpo no decorrer de uma atividade física. A energia para fosforilar a adenosina difosfato (ADP) durante o exercício intenso provém, principalmente, do glicogênio muscular por meio da glicólise anaeróbica, com a subsequente formação de lactato. Quando as células musculares não conseguem oxidar no mesmo ritmo da produção, o acúmulo de lactato aumenta de acordo com o aumento da intensidade do exercício (MCARDLE, 2011).

Existem alguns índices que necessitam de proce- dimento invasivo, responsáveis pela avaliação e quantificação da capacidade aeróbia, que permitem aos profissionais uma forma de acompanhamento do treinamento desportivo. Dentre esses, a resposta da lactacidemia utilizada para determinar o Limiar Anaeróbio (LAn) durante o exercício talvez seja a forma mais precisa de mensuração da capacidade aeróbia, já que apresenta sensibilidade comprovada ao treinamento físico, além de servir como preditor da performance (DENADAI, 1999; DENADAI, 2000).

A resposta do lactato sanguíneo, durante exercícios de cargas crescentes, possibilita a identificação do LAn (SIMÕES, 2000; SIMÕES et al., 1999). Ultimamente, vários protocolos são utilizados para determinação do LAn. Alguns se utilizam de variáveis ventilatórias, enquanto muitos se utilizam de variáveis metabólicas, especialmente dosagens de lactato sanguíneo.

Pinto et al (2014) analisaram que, em exercícios de alta intensidade e curta duração, o acúmulo de íons Hidrogênio $(\mathrm{H}+)$ no interior da célula muscular é um dos principais agentes causadores da fadiga. Este estudo enfatiza que, nesse tipo de atividade, a taxa de ressíntese de ATP pelas vias oxidativas se torna insuficiente para suprir a demanda celular e a principal fonte de ATP passa a ser a glicólise, culminando em um aumento na produção de lactato.

Gentil et al. (2006) mostraram, no seu estudo de comparação dos níveis de lactato em diferentes métodos de treinamento de força (MTF), que, em todos os MTF, os níveis de lactato sanguíneo tiveram aumentos significativos quando comparados com o repouso, contudo, entre os MTF aplicados não houve diferenças nas respostas, sugerindo que todos os métodos parecem fornecer os mesmos estímulos metabólicos.

Arbabi (2015) avaliou, em seu estudo, o uso de compressão de corpo inteiro por um período de 24 h após uma sessão de alta intensidade de treinamento resistido (3 séries de 8-10 RM com halteres e 2-2,5 min de descanso) em 11 voluntários homens (23,0 $\pm 2,9$ anos) e 9 mulheres (23,1 $\pm 2,2$ anos). Por meio dos resultados, o uso de roupas de compressão de corpo inteiro para recuperação resulta em menores índices de fadiga, dor muscular, concentração de creatina quinase (CK) e inchaço muscular e um melhor desempenho no exercício. Não houve diferença estatística para a concentração de lactato desidrogenase.

Segundo Ali, Creasy, Edge (2011), o uso de roupas 
compressivas surgiu com as meias de compressão e tinha como objetivo ajudar a circulação periférica, melhorar o fluxo sanguíneo e aumentar o retorno venoso. Partindo desses benefícios advindos do uso das meias compressivas, vários estudos investigaram os efeitos do uso dessas vestimentas na melhoria de diversas atividades físicas.

Kemmler et al (2009) verificaram, em seus estudos, que o uso de bermudas de compressão melhoram os mecanismos de propriocepção e O’Donnell et al (1979) indicam que há melhorias na redução da oscilação muscular que pode levar ao dano muscular. Esses fatos podem justificar o aumento na concentração lactacidêmica no momento Pós0’ em que os indivíduos conseguiram realizar mais repetições pela melhora na performance e execução do exercício

Kraemer et al. (1998), em seu estudo com 20 praticantes (10 homens $-23,2 \pm 4.8$ anos; height 1,767 $\pm 0,68$ $\mathrm{m} ; 85 \pm 10 \mathrm{~kg} ; 12.8 \pm 9.2 \%$ gordura) e 10 mulheres $-25,2$ $\pm 3,8$ anos; $1,683 \pm 0,108 \mathrm{~m} ; 62 \pm 12 \mathrm{~kg} ; 22,1 \pm 4,2 \%$ gordura), de treinamento resistido, divididos em dois grupos conforme o sexo, analisaram a quantidade máxima possível de repetições no agachamento à $70 \%$ de $1 \mathrm{RM}$, não havendo diferença significativa no número de repetições realizadas, além de não haver nenhuma evidência de que os calções de compressão ajudam na remoção de lactato.

Gentil et al. (2006) definiram a oclusão vascular adaptada como sendo um tipo de contração máxima isométrica de 20 segundos com carga de 10RM acompanhada, sequencialmente, por levantamentos isoinerciais normais, com carga de 10RM, até a falha concêntrica. Esse método, normalmente, é utilizado para conquistar os benefícios da oclusão vascular, uma vez que ações isométricas são relacionadas à diminuição do fluxo sanguíneo e ao aumento dos resíduos metabólicos, sendo correlacionado o aumento dos níveis de lactato com calça de compressão.

O objetivo do presente estudo foi comparar a concentração de lactato sanguíneo no exercício de agachamento com e sem calça compressão.

\section{Materiais e Métodos}

\subsection{Participantes}

Participaram da pesquisa 8 indivíduos do sexo masculino $(n=8)$, conforme Tabela 1 , com faixa etária entre 18 a 30 anos, fisicamente ativos, praticantes de treinamento resistido há pelo menos 6 meses, com duração mínima de 5 horas semanais.
O estudo foi aprovado pelo comitê de ética em pesquisa do Centro Universitário de Brasília - UniCEUB e aprovado pelo CAAE: 48991515.7.0000.0023 e parecer: 1.250.581/2015, em 29 de setembro de 2015. Os voluntários foram informados do objetivo e os procedimentos. Todos os sujeitos forneceram consentimento livre e esclarecido por escrito, e sem nenhuma patologia, conforme Apêndice B. Foi usado como critério de exclusão do estudo história de doença cardiovascular ou doenças osteomioarticulares de qualquer segmento dos membros inferiores, que impediriam a realização do agachamento neste estudo.

\subsection{Materiais utilizados}

Para os dados de caracterização da amostra, utilizou-se a balança mecânica antropométrica FILIZOLA M31 para mensurar a massa corporal, o estadiômetro personal capriche da sanny para mensurar a estatura, o adipômetro científico da sanny para a composição corporal através do protocolo 3 dobras de Pollock, o lactimêtro Accutrend PLUS - Roche para mensuração do lactato, o metrônomo da Pro metrônomo para a cadência do exercício e a calça de compressão Nike tech tight para o exercício de agachamento.

Tabela1. Características dos participantes

\begin{tabular}{lc}
\hline Variável & Média \pm Desvio Padrão \\
\hline Idade (anos) & $21,50 \pm 3,07$ \\
Massa Corporal (kg) & $79,88 \pm 12,26$ \\
Estatura (m) & $1,77 \pm 0,08$ \\
Índice de Massa Corporal $\left(\mathrm{kg} / \mathrm{m}^{2}\right)$ & $25,37 \pm 2,85$ \\
Percentual de Gordura $(\%)$ & $9,22 \pm 3,71$ \\
1Repetição Máxima (kg) & $128,63 \pm 37,91$ \\
Média da carga do teste de 10RM (kg) & $64,31 \pm 18,02$ \\
\hline
\end{tabular}

Fonte: Do autor.

\subsection{Procedimento das coletas - Teste experimental e Coleta de lactato \\ 2.3.1 Primeiro dia}

Foram mensuradas as características antropométricas, como massa corporal e estatura, para calcular o índice de massa corporal e composição corporal. Os dados referentes às características antropométricas são apresentados na tabela 01 . As coletas dos dias 3 e 4 foram randomizadas. 


\subsubsection{Segundo Dia}

Os voluntários foram submetidos a um teste de 10 repetições máximas (10RM), proposto por Baechle e Earle (2000). Para realização desse teste, os voluntários realizaram um aquecimento específico composto por uma série de 15 repetições com carga aproximada de 50\% de 10RM. Após o aquecimento, o voluntário teve 3 tentativas para realizar 10 repetições máximas, com a carga ajustada pelo responsável pela coleta, com intervalo de cinco minutos entre as tentativas. A carga foi considerada válida quando o participante foi capaz de realizá-la de acordo com o protocolo. Os testes foram realizados no laboratório de fisiologia humana do Centro Universitário de Brasília (UniCEUB).

O protocolo de intervenção foi composto por 6 séries de agachamento até 10 repetições máximas, com intervalo de 50 segundos entre as séries a 70\% de 1RM, que foi calculado por meio do teste de predição de Baechle e Earle (2000). Foram instruídos a executar a fase concêntrica e excêntrica do exercício de forma controla$\mathrm{da}$, com velocidade de 2 segundos para ambas as fases, não havendo pausa na transição entre essas duas fases. A velocidade de execução foi controlada com auxílio de um metrônomo digital. O lactato foi mensurado com o objetivo de determinar o nível sérico de Lactato.

\subsubsection{Terceiro dia}

A primeira coleta (Pré) foi feita após cinco minutos de descanso com os voluntários do grupo controle (sem calça de compressão) sentados. A segunda coleta (Pós0') foi feita logo após as 6 séries de treinamento. A terceira coleta (Pós10') foi feita dez minutos depois do teste com os voluntários sentados.

\subsubsection{Quarto dia}

A primeira coleta (Pré) foi feita após cinco minutos de descanso com os voluntários do grupo experimental (com calça de compressão) sentados. A segunda coleta (Pós0') foi feita logo após as 6 séries de treinamento. A terceira coleta (Pós10') foi feita dez minutos depois do teste com os voluntários sentados.

\subsection{Análise Estatística}

A análise descritiva foi utilizada para caracterizar a amostra, os valores de todas as variáveis foram expressos em média e desvio padrão. A normalidade dos dados foi verificada pelo teste de Shapiro-Wilk e foi adotada a estatística paramétrica. A concentração de lactato nas duas condições (controle e experimental) nos três momentos
(Pré, Pós0’ e Pós10’) foram analisadas através de uma ANOVA fatorial de medidas repetidas de dois fatores (intervenção versus momento). O Tratamento de Bonferroni foi utilizado para identificar as diferenças significativas. Todas as análises estatísticas foram realizadas no software estatístico SPSS versão 21.0 (SPSS Inc., Somers, NY, USA). Adotou-se $\mathrm{p} \leq 0,05$ como nível de significância.

\section{Resultados}

A concentração de lactato não apresentou diferença entre as intervenções no momento pré $(\mathrm{p}>0,05)$. A concentração de lactato no momento Pós0' apresentou uma elevação quando comparado com o momento Pré nas duas condições $(\mathrm{p}<0,05)$. No momento Pós10', a concentração de lactato se manteve, significativamente, mais alta $(p<0,05)$ que no repouso apenas na intervenção com calça de compressão. A concentração de lactato no momento Pós0’ da intervenção com a calça de compressão atingiu níveis significativamente mais altos que no controle $(\mathrm{p}<0,05)$. O comportamento do lactato sanguíneo nas duas condições (controle e experimental) está exposto na tabela 2.

Tabela 2. Resposta da concentração de lactato sanguíneo nas duas condições e nos três momentos.

\begin{tabular}{lccc}
\hline Lactato $(\mathrm{mmol} / \mathrm{l})$ & Pré & Pós0' & Pós10' \\
\hline Experimental & $2,80 \pm 0,76$ & $15,10 \pm 1,25^{* \&}$ & $12,64 \pm 1,70^{* \&}$ \\
\hline Controle & $3,21 \pm 0,80$ & $10,41 \pm 1,27^{*}$ & $6,71 \pm 2,88^{\&}$ \\
\hline
\end{tabular}

Fonte: Do autor.

Legenda: * $p<0,05$ em relação ao momento pré; ${ }^{\circledR} p<0,05$ em relação à intervenção sem calça de compressão

\section{Discussão}

Figueiredo, Figueiredo e Silva (2011), no estudo feito com 10 jogadoras profissionais de voleibol, com idade entre 18 e 25 anos e sem lesões, usando meias de compressão, obteve os seguintes resultados de lactato, em valores médios: $134,2 \pm 11,3 \mathrm{U} / \mathrm{L}$ em M0 (em repouso);

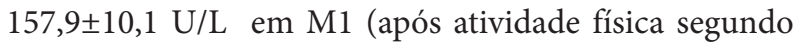
o protocolo - alongamento, aquecimento com movimentos típicos do esporte e sprints de $30 \mathrm{~m}$ com $15 \mathrm{~s}$ de descanso - com uso da meia de compressão); e 177 $\pm 18,8$ U/L, em M2 (após atividade física sem uso da meia de compressão), mostrando uma diminuição nos níveis de lactato desidrogenasse (LDH) quando em uso da meia de compressão, com diferenças estatisticamente significati- 
vas entre M1 e M2 ( $\mathrm{p}=0,0000)$. No entanto, o presente estudo, comparando a concentração de lactato utilizando e não utilizando a calça de compressão durante o exercício de agachamento, os níveis de foram menores no grupo controle $(6,71 \pm 2,88)$ quando comparados ao grupo experimental $(12,64 \pm 1,70)$, com o valor de $(\mathrm{p}<0,05)$.

Kraemer et al. (2010) mostraram, em seu estudo com praticantes de treinamento resistido, que não há nenhuma evidência de que os calções de compressão ajudam na remoção de lactato, além de não haver diferença significativa na quantidade de repetições realizadas no agachamento em um teste de resistência (homens com bermuda: 32 repetições, controle: 29 repetições e mulheres com bermuda: 30 repetições, controle: 31 repetições) e nem no trabalho durante um teste de flexão $(p<0,05)$ e extensão $(\mathrm{p}<0,05)$. No presente estudo, os níveis de lactato mantiveram-se significativamente mais altos no grupo experimental no momento Pós0’ e Pós10', não havendo diminuição nos níveis de lactato comparando com o grupo controle.

A compactação ideal da roupa de compressão reduz a quantidade do movimento e a oscilação durante o movimento, além de permitir que haja uma reparação dos tecidos dos membros mantidos em posição anatômica que não estão sendo recrutados durante o exercício. A roupa de compressão, também, tem sido relacionada com o aumento da bomba muscular, que permite o retorno do sangue ao coração, acontecendo a troca do sangue venoso pelo sangue rico em oxigênio, removendo os produtos residuais (ARBABI, 2015). No presente estudo, a análise Pós10' apresentou concentração de lactato mais alta na intervenção com calça $(12,64 \pm 1,70)$ quando comparada com o controle $(6,71 \pm 2,88)$, mostrando que a calça de compressão não influenciou a redução dos níveis de lactato na amostra estudada.

No estudo de Maton et al (2006), os resultados indicaram que o uso de compressão em membros inferiores podem moderar a perda de força durante a execução e a percepção de dor muscular tardia, justificando a eficiência nas repetições.

Gentil et al. (2006) usaram, em seu estudo, um método chamado oclusão vascular adaptada, que é um tipo de contração máxima isométrica de 20 segundos com carga de 10RM, seguida por levantamentos isoinerciais normais com carga de 10RM, até falha concêntrica. Esse método, normalmente, é utilizado para conquistar os benefícios da oclusão vascular, uma vez que ações isomé- tricas são relacionadas a diminuição do fluxo sanguíneo e aumento dos resíduos metabólicos. No presente estudo, os níveis de lactato foram maiores no grupo com calça de compressão, comparado com o grupo controle, nas coletas Pós0' e Pós10', podendo ter relação com a oclusão que a calça transfere à superfície corporal, trazendo maiores níveis de lactato para o grupo.

\section{Conclusões}

Em decorrência do universo amostral restrito do presente estudo, não foi possível chegar à conclusão confirmatória que o uso da calça de compressão figura como fator determinante para o incremento da atividade física agachamento, referente aos níveis de lactato sanguíneo, uma vez que a concentração de lactato se manteve, significativamente, elevada após dez minutos da intervenção em relação ao grupo controle pós-exercício. Assim, a utilização isolada da calça de compressão não é a melhor solução que se coaduna com a expectativa de obtenção de níveis de lactato sanguíneo mais baixos após o exercício de agachamento.

\section{Referências}

ALI, A.; CREASY, R. H.; EDGE, J. A. The effect of graduated compression stockings on running performance. Journal of Strength and Conditioning Research, Champaign, v. 25, n. 5, p. 1385-1392, May 2011. doi: 10.1519/ JSC.0b013e3181d6848e

ARBABI, A. Effects of a whole body compression garment on recovery after a heavy resistance. Turkish Journal of Sport and Exercise, Konya, v. 17, n. 1, p. 43-51, Jan. 2015.

BAECHLE, T. R.; EARLE, R.W. Essential of Strength Training and Conditioning. Champaign: Human Kinetics, 2000.

DENADAI, B. S. Avaliação aeróbia: consumo máximo de oxigênio ou resposta do lactato sanguíneo? In: DENADAI, B. S. Avaliação aeróbia: determinação indireta da resposta do lactato sanguíneo. Rio Claro: Motrix, 2000. p. 3-24.

DENADAI, B. S. Índices fisiológicos de avaliação aeróbia: conceitos e aplicações. Ribeirão Preto: BSD, 1999.

FIGUEIREDO, M. A. M.; CASTRO, A. A.; SIMŌES, R. Terapia de compressão de membros inferiores. 2011. Disponível em: <https://diretrizes.amb.org.br/_BibliotecaAntiga/terapia_de_compressao_de_membros_inferiores.pdf>. Acesso em: 23 mar. 2017. 
FIGUEIREDO, M.; FIGUEIREDO, M. F; SILVA, N. P. Efeito do uso de meia elástica sobre os níveis dos biomarcadores de lesão muscular em atletas de voleibol após atividade física. Jornal Vascular Brasileiro, Porto Alegre, v. 10, n. 4, p. 289-292, dez. 2011. doi: 10.1590/S167754492011000400005

GENTIL, P. et al. Efeitos agudos de vários métodos de treinamento de força no lactato sanguíneo e características de cargas em homens treinados recreacionalmente. Revista Brasileira de Medicina do Esporte, Niterói, v. 12, n. 6, p. 303-307, nov./dez. 2006. doi:10.1590/S151786922006000600001 .

KEMMLER, W. et al. Effect of compression stockings on running performance in men runners. Journal of Strength and Conditioning Research, Champaign, v. 23, n. 1, p. 101-105, jan. 2009. doi: 10.1519/ JSC.0b013e31818eaef3.

KRAEMER, W. J. et al. Compression garments: Influence on muscle fatigue. Journal of Strength \& Conditioning Research, Champaign, v.12, n. 4, p. 211-215, nov. 1998.

KRAEMER, W. J. et al. Effects of a whole body compression garment on markers of recovery after a heavy resistance workout in men and women. Journal of Strength \& Conditioning Research, Champaign, v. 24, n. 3, p. 804814, mar. 2010. doi: 10.1519/JSC.0b013e3181d33025.

MATON, B. et al. Human muscle fatigue and elastic compressive stockings. European Journal of Applied Physiology, Berlin, v. 97, n. 4, p. 432-442, Jul. 2006. doi:10.1007/s00421-006-0187-8
MCARDLE, W. Transferência de Energia no Exercício. In: KATCH, V. L.; KATCH, F. I.; MCARDLE, W. D. Fisiologia do exercício: Energia, Nutrição e Desempenho Humano. 6. ed. Rio de Janeiro: Guanabara, 2011. p. 167-182.

O'DONNELL JUNIOR, T. F. et al. Effect of elastic compression on venous hemodynamics in postphlebitic limbs. JAMA, Chicago, v. 242, n. 25, p. 2766-2768, Dec. 1979. doi:10.1001/jama.1979.03300250022022

PINTO, C. L. et al. Lactato: de causa da fadiga a suplemento ergogênico? Revista Brasileira de Ciência e Movimento, Brasília, v. 22, n. 2, p. 173-181, fev. 2014. doi:10.18511/0103-1716/rbcm.v22n2p173-181

SEAR, J. A. et al. The effects of whole-body compression garments on prolonged high-intensity intermittent exercise. Journal of Strength \& Conditioning Research, Champaign, v. 24, n. 7, p. 1901-1910, Jul. 2010. doi: 10.1519/JSC.0b013e3181db251b.

SIMÕES, H.G. Respostas metabólicas e hormonais durantes os testes de determinação do limiar anaeróbio individual e lactato mínimo. 2000. 186 f. Tese (Doutorado) - Centro de Ciências Biológicas e da Saúde. Universidade Federal de São Carlos, São Carlos, 2000.

SIMÕES, H. G. et al. Blood glucose responses in humans mirror lactate responses for individual anaerobic threshold and for lactate minimum in track tests. European Journal of Applied Physiology and Occupational Physiology, Berlin, v. 80, n. 1, p. 34-40, May 1999. doi:10.1007/ s004210050555. 